\title{
Dimensional Crossover in Two-Dimensional Bose-Fermi Mixtures ${ }^{1}$
}

\author{
A. L. Subaşii ${ }^{a}$, S. Sevinçli ${ }^{b}$, P. Vignolo ${ }^{c}$, and B. Tanatar ${ }^{a}, *$ \\ ${ }^{a}$ Department of Physics, Bilkent University, Bilkent, Ankara 06800, Turkey \\ ${ }^{b}$ Max-Planck-Institut für Physik komplexer Systeme, Nöthnitzer Str. 38, 01187 Dresden, Germany \\ ${ }^{c}$ Institut Non Linéaire de Nice, Univeraité de Nice-Sophia Antipolis, CNRS, \\ 1361 route de Lucioles, 06560 Valbonne, France \\ *e-mail: tanatar@fen.bilkent.edu.tr \\ Received October 15, 2009; published online February 2, 2010
}

\begin{abstract}
We investigate the equilibrium properties of boson-fermion mixtures consisting of a Bose condensate and spin-polarized Fermi gas confined in a harmonic two-dimensional (2D) trap using mean-field theory. Boson-boson and boson-fermion coupling constants have a logarithmic dependence on the density because of the two-dimensional scattering events when the $s$-wave scattering lengths are on the order of mixture thickness. We show that this modifies the density profiles significantly. It is also shown that the dimensional crossover stabilizes the mixture against collapse and spatial demixing is observed for the case of a negative boson-fermion scattering length.
\end{abstract}

DOI: $10.1134 / \mathrm{S} 1054660 \mathrm{X} 1005018 \mathrm{X}$

\section{INTRODUCTION}

Recently, quantum degenerate atomic mixtures have been studied extensively both in theoretical and experimental directions. Fermionic degeneracy has been observed by means of sympathetic cooling with many alkali atom mixtures like ${ }^{7} \mathrm{Li}-{ }^{6} \mathrm{Li}[1,2],{ }^{23} \mathrm{Na}-$ ${ }^{6} \mathrm{Li}[3],{ }^{87} \mathrm{Rb}-{ }^{40} \mathrm{~K}$ [4-6], and recently with an isotopic mixture of ytterbium $(\mathrm{Yb}),{ }^{174} \mathrm{Yb}-{ }^{173} \mathrm{Yb}$ [7]. Interaction between the different species strongly affects the equilibrium properties of the mixture such that the mixture collapses [6] in the presence of attractive boson-fermion coupling, or in the opposite case, repulsion between the species causes spatial demixing $[8,9]$.

Boson-fermion (BF) mixtures can also be realized from an imbalanced two-component Fermi gas where bound fermions form a Bose-Einstein condensate (BEC). However, BF mixtures of two atomic species have the advantage that the interaction between the bosons and between the different species can be driven independently and attractive BF interaction is accessible $[10,11]$.

The properties of three-dimensional (3D) trapped BF mixtures were studied in detail by using a modified Gross-Pitaevskii (GP) equation [12, 13], and by using the Thomas-Fermi (TF) approximation for the fermionic component $[14,15]$. In a quasi-3D limit, where collisions can still be considered as $3 \mathrm{D}$, effects of the geometry were also studied in the TF regime [16]. Such a simple model predicts, in a pancake-

\footnotetext{
${ }^{1}$ The article is published in the original.
}

shaped trap, that the stability of the mixture depends only on the scattering length and the transverse width of the cloud. One should expect, in a true dimensional crossover, namely including dimensional effects in scattering events, that the mixture stability depends critically on the energy, and thus on the number of particles. There are also studies on BF mixtures in two-dimensional (2D) [17] and one-dimensional (1D) [18] optical lattices in which many-body phase diagrams has been obtained by the bosonization approach.

It is well known that a hard-core boson gas shows very different features from its $3 \mathrm{D}$ counterpart if the scattering-events are two-dimensional. In 3D, particle interactions can be described by the zero-momentum and zero-energy limit of $T$-matrix, leading to a constant coupling parameter. In $2 \mathrm{D}$, the $T$-matrix vanishes at low momentum and energy $[19,20]$ and the first-order contribution to the coupling is obtained by taking into account the many-body shift in the effective collision energy of two-condensate atoms [21, 22]. This causes an energy dependent coupling parameter which greatly affects the equilibrium and the dynamical properties of the gas [23, 24].

The dimensional crossover from a $3 \mathrm{D}$ to a $2 \mathrm{D}$ trapped mixture may be studied in the experiments by flattening magnetic or dipolar confinements [25], or by trapping atoms in specially designed pancake potentials, as rotating traps [26], gravito-optical surface traps [27], rf-induced two-dimensional traps [28] or in one-dimensional lattices [29] where a 3D gas can be split in several independent disks. 
In this paper, the equilibrium properties of a mixture of condensed bosons and spin-polarized fermions are studied, through the dimensional crossover from three to two dimensions. There is no fermion-fermion interaction and we include the BF $s$-wave interaction self-consistently in a suitably modified GP equation for bosons [30]. The chemical potentials and the radii of the clouds are also obtained by using the TF approximation.

\section{MEAN-FIELD MODEL}

We consider a $2 \mathrm{D} \mathrm{BF}$ mixture in harmonic trap with potentials $V_{B(F)}=\frac{1}{2} m_{B(F)} \omega_{B(F)}^{2} r^{2}$ and particle numbers $N_{B}$ and $N_{F}$. Here, $m_{B(F)}$ is the boson (fermion) mass and $\omega_{B(F)}$ is the radial trap frequency as seen by boson (fermion) species. Within the mean-field approach the total energy functional at $T=0$ is written as

$$
\begin{gathered}
E\left[\psi_{B}, \psi_{F}\right] \\
=\int d^{2} r\left\{\frac{\hbar^{2}}{2 m_{B}}\left|\nabla \psi_{B}\right|^{2}+V_{B}(r)\left|\psi_{B}\right|^{2}+\frac{1}{2} g_{B B}\left|\psi_{B}\right|^{4}\right\} \\
+\int d^{2} r\left\{T_{F}+V_{F}(r)\left|\psi_{F}\right|^{2}\right\}+\int d^{2} r g_{B F}\left|\psi_{B}\right|^{2}\left|\psi_{F}\right|^{2},
\end{gathered}
$$

where $\psi_{B}$ is the ground-state wave function of bosons and $\left|\psi_{F}\right|^{2}=n_{F}$ gives the fermion density. In the above expression, boson species are in the condensed state and fermion component is assumed to be spin-polarized, there is no fermion-fermion interaction, and $g_{B B}$ and $g_{B F}$ are the interaction couplings between the bosons and between bosons and fermions, respectively. There is a significant difference between the form of the energy functional given above and that in $3 \mathrm{D}$, which is that the $\mathrm{BB}$ and $\mathrm{BF}$ interaction strengths are in general density dependent in contrast to the situation in $3 \mathrm{D}$. The term $T_{F}$ in Eq. (1) is the kinetic energy of the fermions written within the Thomas-FermiWeizacker approximation as [31-33]

$$
T_{F}=\frac{\hbar^{2}}{m_{F}}\left(\pi n_{F}^{2}+\frac{\lambda_{W}}{8} \frac{\left|\nabla n_{F}\right|^{2}}{n_{F}}\right),
$$

where the Weizsacker constant is $\lambda_{W}=1 / 4$. The Euler-Lagrange equations for the mixture are $[32,33]$

$$
\begin{gathered}
\frac{\partial \mathscr{F}_{F}}{\partial \psi_{B}}-\nabla \frac{\partial \mathscr{F}_{F}}{\partial\left(\nabla \psi_{B}\right)}=0 \\
\text { and } \frac{\partial \mathscr{F}_{F}}{\partial \psi_{F}}-\nabla \frac{\partial \mathscr{F}_{F}}{\partial\left(\nabla \psi_{F}\right)}=0,
\end{gathered}
$$

where $\mathscr{F}=\mathscr{E}-\mu_{B}\left|\psi_{B}\right|^{2}-\mu_{F}\left|\psi_{F}\right|^{2}$ with $\mu_{B}$ and $\mu_{F}$, the boson and fermion chemical potential entering as Lagrange multipliers to satisfy the normalization con- ditions $N_{B}=\int d^{2} r\left|\psi_{B}\right|^{2}$ and $N_{F}=\int d^{2} r\left|\psi_{F}\right|^{2}$. Then, the explicit form of the equations of motion are

$$
\left\{-\frac{\hbar^{2}}{2 m_{B}} \nabla^{2}+V_{B}+g_{B B}\left|\psi_{B}\right|^{2}+g_{B F}\left|\psi_{F}\right|^{2}-\mu_{B}\right\} \psi_{B}=0
$$

and

$$
\begin{gathered}
\left\{-\frac{\hbar^{2}}{2 m_{F}} \lambda_{W} \nabla^{2}+V_{F}\right. \\
\left.+\frac{\hbar^{2}}{m_{F}} 2 \pi\left|\psi_{F}\right|^{2}+g_{B F}\left|\psi_{B}\right|^{2}-\mu_{F}\right\} \psi_{F}=0 .
\end{gathered}
$$

The above equations of motion are obtained by neglecting the higher-order terms involving $\delta g / \delta \psi_{B, F}$ which is valid in the dilute gas limit $n_{B} a_{B B}^{2} \ll 1$ and $n_{F} a_{B F}^{2} \ll 1$. The beyond mean-field corrections can become notable when $a_{B B}, a_{B F}$ and/or $N_{B}, N_{F}$ are large for fixed trap frequencies. For the systems under consideration we have chosen the parameters appropriately so the diluteness conditions hold for our numerical calculations. Therefore, the beyond mean-field terms in the energy functional are not important in our examples.

One should also note that the existence of BEC in 2D needs to be examined carefully. Initial studies have concluded that no BEC could occur in 2D trapped gases but recent considerations within the HartreeFock-Bogoliubov approximation [34], the density dependent interaction strength [35] and numerical simulations [36] have established firmly the occurrence of BEC for such systems. Thus, our assumption of a $2 \mathrm{D}$ condensate at $T=0$ is justified.

When the number of atoms is large one can use the Thomas-Fermi (TF) approximation, i.e., the kinetic energy terms can be neglected in the GP equations, which simplifies them to coupled algebraic equations

$$
\begin{aligned}
& g_{B B}\left|\psi_{B}\right|^{2}+g_{B F}\left|\psi_{F}\right|^{2}+V_{B}=\mu_{B}, \\
& g_{F F}\left|\psi_{F}\right|^{2}+g_{B F}\left|\psi_{B}\right|^{2}+V_{F}=\mu_{F} .
\end{aligned}
$$

In the above, $\left|\psi_{B}\right|^{2},\left|\psi_{F}\right|^{2} \geq 0$ and we have defined the effective fermion-fermion interaction coupling $g_{F F}=$ $2 \pi \hbar^{2} / m_{F}$. We have also introduced the TF radii $R_{B}, R_{F}$ where boson and fermion wave functions go to zero respectively through $\left|\psi_{B}\left(R_{B}\right)\right|^{2}=0$ and $\left|\psi_{F}\left(R_{F}\right)\right|^{2}=0$. Assuming $R_{F} \geq R_{B}$ (since $g_{F F} \gg g_{B B}$, fermions are pushed out further due to Pauli exclusion principle) 